\title{
COMUNIDADES DE LOMBRICES DE TIERRA EN SISTEMAS AGROFORESTALES INTERCALADOS, EN DOS REGIONES DEL CENTRO DE MEXICO
}

\author{
DIONICIO JUÁREZ-RAMÓN ${ }^{1}$ Y CARLOS FRAGOSO ${ }^{2}$ \\ ${ }^{1}$ Centro de Agroecología, Instituto de Ciencias, Benemérita Universidad Autónoma de Puebla, Blvd. \\ 14 sur 6301 Ciudad Universitaria, C.P. 72570 Puebla, Puebla, México. < dionicio.juarez@correo.buap. \\ $\mathrm{mx}>$ \\ ${ }^{2}$ Red de Biodiversidad y Sistemática, Instituto de Ecología, A.C., Km. 2.5 Car. Antigua a Coatepec \\ 351, Congregación El Haya, Xalapa 91070, Veracruz, México. < carlos.fragoso@inecol.mx>
}

Juárez-Ramón, D. y Fragoso, C. 2014. Comunidades de lombrices de tierra en sistemas agroforestales intercalados, en dos regiones del centro de México. Acta Zoológica Mexicana (n.s.), 30(3): 637-654.

RESUMEN. Se realizó un análisis de las comunidades de lombrices de tierra del sistema de cultivo milpa intercalada con árboles frutales (MIAF), en los estados de Puebla y Oaxaca. El objetivo fue conocer las especies presentes en el sistema, las diferencias entre un bosque control y los sistemas MIAF, y al interior de este entre la zona de milpa y la arbórea. En el estudio se calculó la abundancia, biomasa y diversidad de especies epigeas y endogeas. En los sistemas MIAF de Puebla se encontraron cuatro especies (una epigea y tres endogeas), mientras que en Oaxaca fueron cinco (dos epigeas y tres endógeas). En Puebla el 64\% de las lombrices se concentraron en el bosque, siendo Lumbricus rubellus, Hoffmeister, 1843 y Aporrectodea trapezoides (Dugès, 1828), las dominantes; en Oaxaca el 79\% de los individuos se encontraron en las áreas cultivadas, dominando las especies Octolasion tyrtaeum (Savigny, 1826), Amynthas sp., y Pontoscolex corethrurus (Müller, 1857). Las diferencias en la abundancia, la biomasa y la cantidad y clase de especies estarían relacionadas con la humedad del suelo, la intensificación agrícola y el manejo antropogénico de los materiales orgánicos (desechos de cosechas y estiércoles). Los resultados indicaron que las líneas de árboles frutales no presentaron comunidades de lombrices de tierra más diversas y abundantes, aunque esto fue más notorio en la región de Puebla. En ninguna de las dos regiones de estudio la abundancia total de lombrices alcanzó los $30 \mathrm{~g} \mathrm{~m}^{-2}$ recomendados para impactar en la fertilidad del suelo, por lo que se sugiere implementar técnicas de manejo en los sistemas MIAF que incrementen la cantidad de lombrices de talla mediana o grande.

Palabras clave: Lombrices de tierra, durazno, MIAF, sequía.

Juárez-Ramón, D. \& Fragoso, C. 2014. Earthworm communities in intercropping systems, from two regions of central Mexico. Acta Zoológica Mexicana (n.s.), 30(3): 637-654.

ABSTRACT. The study of earthworm communities in an intercropping system (MIAF) was carried out in the central and southern states of Puebla and Oaxaca, Mexico. The aim was to know the species pres-

Recibido: 28/05/2014; aceptado: 27/06/2014. 
ent in MIAF systems, to identify differences between a control nearby forest and MIAF's, as well as differences within MIAF's between arboreal and crop rows. The study evaluated the abundance, biomass and diversity of epigeic and endogeic species. In Puebla four species were found in MIAF's (one epigeic and three endogeics), while in Oaxaca there were five species (two epigeics and three endogeics). In Puebla, $64 \%$ of the worms were concentrated in the forest, being Lumbricus rubellus Hoffmeister, 1843 and Aporrectodea trapezoides (Dugès, 1828), the dominant species; in Oaxaca, 79\% of the individuals were found in MIAF's, being Octolasion tyrtaeum (Savigny, 1826), Amynthas sp., and Pontoscolex corethrurus (Müller, 1857) the dominant species. Differences in species abundance and biomass may be related to soil moisture, agricultural intensification and the anthropogenic management of organic materials (crop residues and manure). Our results indicated that earthworms found in tree rows were not more abundant and diverse than those of annual crop rows; this pattern being more evident in Puebla. Neither in Puebla nor in Oaxaca, the biomass of worms reached $30 \mathrm{~g} \mathrm{~m}^{-2}$, a value where earthworms impact the fertility of soils. Thus it is suggested to improve management techniques within MIAF's in order to increase the abundance of large worms.

Key words: Earthworms, peach, MIAF, Alley cropping, drought.

\section{INTRODUCCIÓN}

Las comunidades de lombrices de tierra (Crasiclitellata, Annelida) son muy importantes en la dinámica edáfica, dado que con sus actividades influyen directa e indirectamente en procesos edáficos como la incorporación de hojarasca al suelo, la descomposición de los materiales orgánicos, la aireación y el reciclaje de nutrientes, entre otros (Werner 1990, Lavelle et al. 1998). Por esta razón han sido ampliamente estudiadas en terrenos cultivados, tanto de climas tropicales (Lavelle et al.1999, Brown et al. 1999) como templados (Hendrix \& Edwards 2004). Su efecto benéfico ha llevado inclusive a la introducción deliberada de algunas especies para rehabilitar y mejorar la fertilidad de los suelos, práctica común en los países templados y fríos y recientemente implementada en los trópicos (Lavelle et al. 1998, Senapati et al. 1999). Además la presencia de lombrices de tierra también puede ser un indicador de la calidad del suelo, ya que su diversidad, densidad y biomasa son sensibles a los cambios de uso del suelo, a la intensificación del cultivo, al uso de maquinaria agrícola y a la aplicación de insumos externos (Mijangos et al. 2006).

Por otro lado el sistema de cultivo milpa intercalada con árboles frutales (MIAF) es un tipo de manejo agroforestal, parecido a otras técnicas de cultivos intercalados llamadas alley cropping en inglés que ha sido rediseñado y tecnificado; este tipo de manejo agroforestal incluye también algunas prácticas de origen prehispánico. En este manejo los surcos de árboles frutales ocupan entre el 33\% y el $42 \%$ del terreno a cultivar, dependiendo si son áreas planas o laderas, respectivamente (Cortés et al. 2004, 2005); el resto de la superficie se destina al cultivo de plantas anuales. Por estos atributos, el MIAF puede ser propicio para la conservación de la biota del suelo incluyendo a las lombrices de tierra, debido a que el área ocupada por los árboles, no es afectada por las labores de labranza convencional. Existen numerosas evidencias que demuestran el efecto negativo de la agricultura convencional sobre la biota del suelo, 
ya sea por prácticas de intensificación agrícola (Postma-Blaauw et al. 2010) o por el arreglo topológico de las especies en el cultivo (Price \& Gordon 1999, Montoya \& Solé 2002).

En México no se han caracterizado en los sistemas MIAF a las comunidades de lombrices de tierra, por lo que en este estudio se pretende identificar a las especies de lombrices y describir los atributos de estas comunidades en dos localidades con diferente clima (templado seco -con riego- vs. templado húmedo) de los estados de Puebla y Oaxaca, respectivamente, en donde se utiliza este sistema MIAF. También se evaluarán los patrones de las comunidades entre las franjas de plantas anuales y las franjas de árboles. Los resultados de este estudio permitirán identificar el efecto de esta práctica de cultivo sobre las comunidades de lombrices de tierra.

\section{MATERIAL Y MÉTODOS}

Sitio de estudio y características edáficas, climáticas y de manejo. Esta investigación se llevó a cabo en parcelas MIAF de campesinos manejadas bajo el sistema intercalado Milpa (maíz, alfalfa, chile, gladiola o frijol) - Durazno, en la región de Chiautzingo y Huejotzingo, Puebla y la región Mazateca en San Jerónimo Tecoatl, Oaxaca. En cada una de las regiones se seleccionaron cuatro MIAFs de diferentes campesinos, y como control una área de bosque (BP - Puebla, y BO - Oaxaca) cercana y representativa de la vegetación original de cada región. Los datos sobre la ubicación y otras características ambientales y de manejo de las dos regiones y de cada uno de los MIAFs se presentan en el Cuadro 1.

Tanto en Puebla (sitios en terreno plano) como en Oaxaca (sitios de ladera) el manejo de los MIAF estuvo orientado a obtener un mismo nivel de rendimientos equivalentes a un promedio de $14.03 \mathrm{Kg}$ de fruta árbol-1 (Torres et al. 2008), a través de paquetes tecnológicos desarrollados por el Colegio de Postgraduados y el Instituto Nacional de Investigaciones Forestales, Agrícolas y Pecuarias (Cortés et al. 2004, 2005). Este manejo incluyó principalmente podas y aplicación de fertilizantes y pesticidas. A pesar de esto, en cada región se observaron diferencias en el manejo de los materiales orgánicos y de la forma e intensidad de remoción del suelo. En Puebla en el sitio P3 se observó la mayor extracción de desechos orgánicos, mientras que los sitios P1, P2 y P4 recibieron aplicaciones esporádicas de estiércol de bovino, sobre todo en los espacios con cultivos anuales; en P4, además, se aplicó anualmente sobre el suelo dedicado a los árboles frutales una capa de $10 \mathrm{~cm}$ de una mezcla de vainas de frijol con rastrojo de maíz (de 1994 al año 2000).

En Oaxaca en todos los sitios se construyeron filtros de escurrimiento perpendiculares a la pendiente para el control de la erosión del suelo, mediante la acumulación en la base de los árboles de durazno, de los restos de las limpias, podas y residuos de las cosechas; en los sitios P6 y P8 el suelo fue más arcilloso y durante el muestreo la parte dedicada a anuales se encontró en descanso; en los sitios P5 y P7, los suelos 


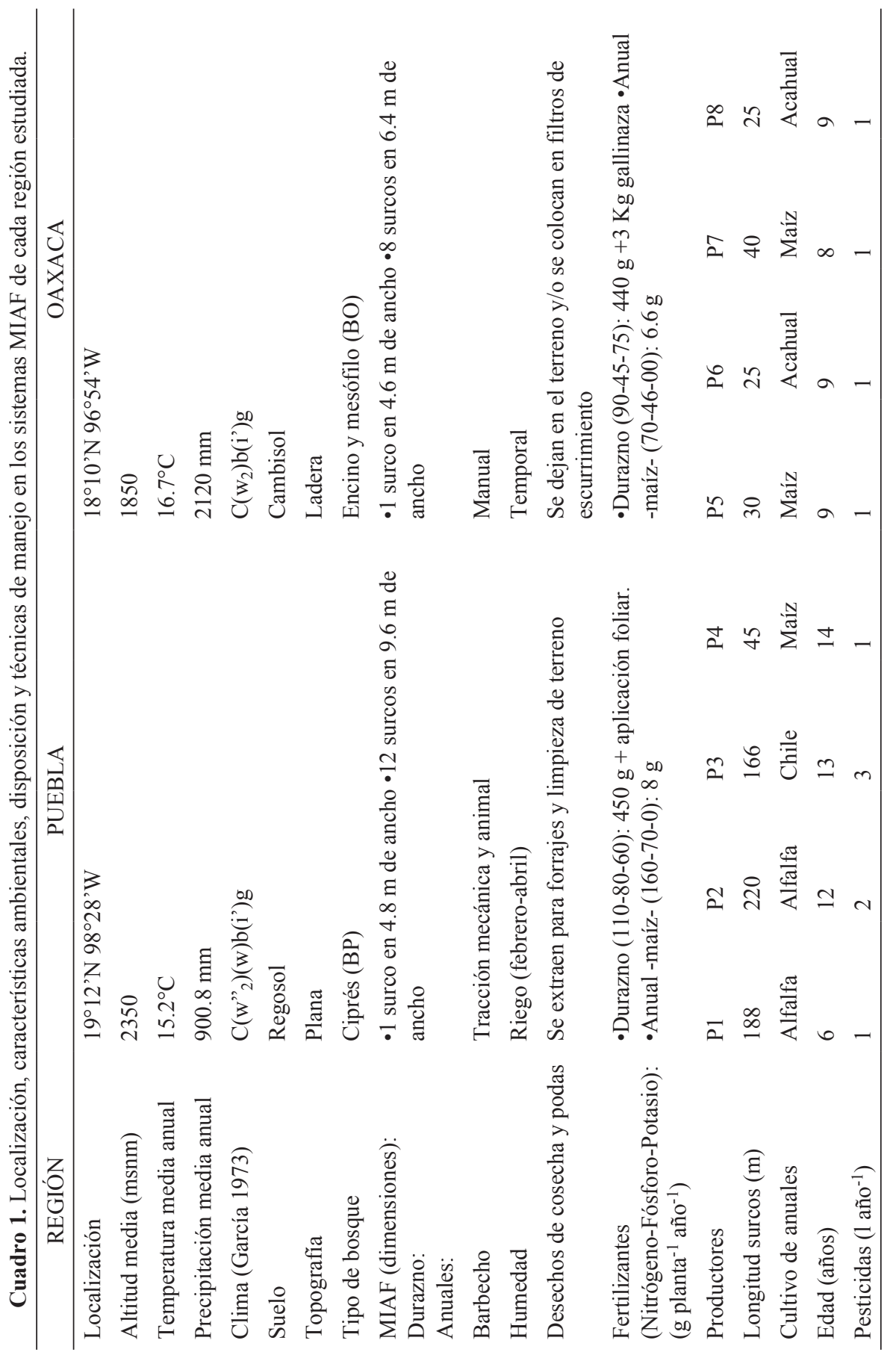


fueron más francos, presentándose un cultivo continuo de maíz por varios años. En este último sitio los filtros de escurrimiento presentaron mayor cantidad de materiales orgánicos acumulados.

Muestreo de las comunidades de lombrices de tierra. En cada uno de los ocho sitios (cuatro en Puebla y cuatro en Oaxaca) y en el bosque correspondiente, se muestrearon las lombrices de tierra al final de la temporada de lluvias (del 30 de octubre al 5 de diciembre del 2006). En cada sitio se ubicaron tres puntos de muestreo repartidos proporcionalmente a lo largo de la línea ocupada por árboles frutales y otros tres sobre la franja ocupada por especies anuales. En los bosques el muestreo se realizó en seis monolitos distanciados a $5 \mathrm{~m}$ cada uno, a lo largo de un transecto de $30 \mathrm{~m}$.

En cada punto de muestreo se realizó un monolito de $25 \times 25 \times 30 \mathrm{~cm}$ de profundidad (método TSBF; Anderson e Ingram 1993), de donde se extrajeron manualmente las lombrices y se fijaron en solución de formol al 4\%; posteriormente se cambiaron a una solución de etanol al 85\% para su separación, cuantificación y pesado. Su identificación se realizó siguiendo las claves de Reynolds (1977) y Blakemore (2006).

Análisis fisicoquímicos. De la misma tierra de cada monolito se separó una muestra de suelo para realizar los siguientes análisis fisicoquímicos: $\mathrm{pH}$ (agua, 1:2), textura (Boyoucos), materia orgánica (Walkey-Black), carbono, nitrógeno total (Kjeldahl), fósforo extractable (Bray), cationes intercambiables $(\mathrm{K}, \mathrm{Ca}, \mathrm{Mg}$ ) y capacidad de intercambio catiónico (CIC), los cuales fueron llevados a cabo en el laboratorio de Alta Resolución (LAAR) del Instituto de Ecología A.C.

Análisis estadísticos. Los datos obtenidos fueron analizados mediante ANOVAS de una vía, previa prueba de homogeneidad de varianzas en cada variable. En los casos en que los supuestos no se cumplían, los datos fueron transformados a rangos, ya que por este método los resultados son más robustos que las pruebas no paramétricas (Conover \& Iman 1981). Las comparaciones de medias fueron estimadas usando Tukey ( $p<0.05$ ) (Zar 2009); asimismo, se analizaron las correlaciones entre variables biológicas y fisicoquímicas, considerando sólo aquellos valores de $\mathrm{R}^{2}$ mayores a 0.5 , que en todos los casos resultaron ser significativos $(\mathrm{p}<0.05)$. En todos los análisis se usó el programa Statistica 6.0.

\section{RESULTADOS}

Especies. En los sitios MIAF de Puebla se registraron cuatro especies de lombrices de tierra (Cuadro 2): tres endogeas (Aporrectodea trapezoides, Octolasion tyrtaeum, Phoenicodrilus taste) y una epigea (Lumbricus rubellus). A. trapezoides y L. rubellus se encontraron tanto en los MIAF como en el bosque, mientras que O. tyrtaeum sólo estuvo en bosque y $P$. taste, sólo en los MIAF.

En los sitios de Oaxaca se registraron cinco especies: tres endogeas (Ocnerodrilus occidentalis, Pontoscolex corethrurus, Octolasion tyrtaeum) y dos epigeas (Amyn- 
Juárez-Ramón y Fragoso: Lombrices de tierra en sistemas agroforestales intercalados

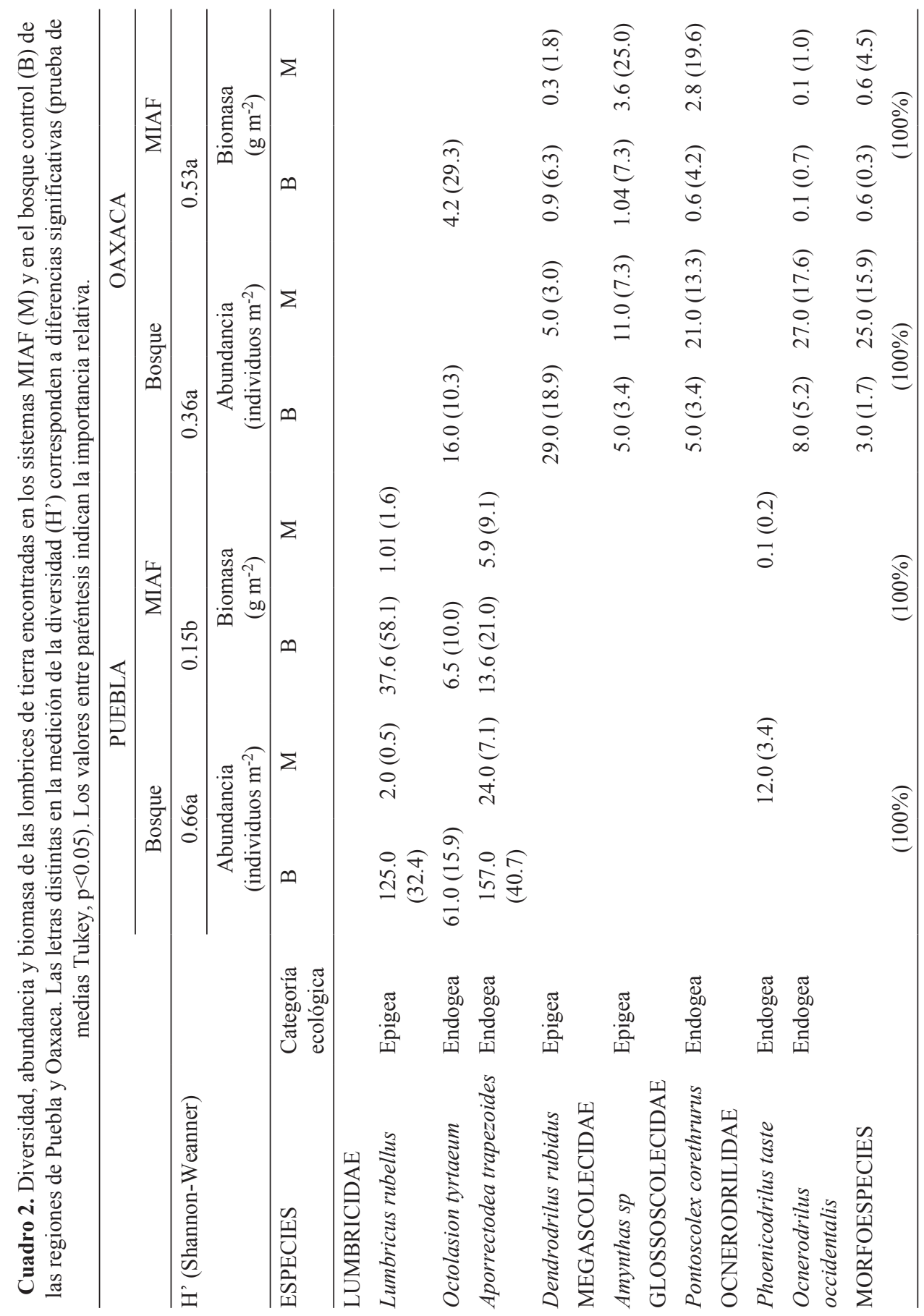


thas sp. y Dendrodrilus rubidus). En las áreas cultivadas estuvo ausente O. tyrtaeum, aunque ésta fue la única especie común entre ambas regiones.

Un grupo de especies de Oaxaca no lograron ser identificadas, por lo que se agruparon como morfo-especies (Cuadro 2).

Todas las especies fueron exóticas, siendo la única excepción $P$. taste, aunque es posible que esta última especie peregrina no sea nativa (Fragoso \& Rojas 2014); de confirmarse esto, entonces todas las especies encontradas serían exóticas.

Comunidades de lombrices en el MIAF. Mientras que en Puebla hubo diferencias en la abundancia $(\mathrm{F}(2,27)=12.14 ; \mathrm{p}<0.00)$ y en la biomasa de lombrices $(\mathrm{F}(2,27)=$ 13.04; $<$ 0.00) entre el bosque y los sitios MIAF, en Oaxaca no se observó este patrón $(\mathrm{F}(2,27)=3.19 ; \mathrm{p}<0.06$ y $\mathrm{F}(2,27)=0.62 ; \mathrm{p}<0.54$, respectivamente $)$. En los bosques de Puebla la abundancia y la biomasa de lombrices fue, respectivamente, nueve y ocho veces mayor que en los sitios MIAF. Al interior de los MIAF no hubo diferencias en ninguna de las dos localidades, al comparar entre las líneas de árboles de duraznos y los cultivos anuales (Fig. 1).

En la diversidad de especies de lombrices se observó el mismo patrón anterior, es decir solamente en Puebla hubo diferencias significativas entre los sitios MIAF y el bosque (Cuadro 2), con una diversidad casi cuatro veces mayor en el bosque; además, los sitios MIAF poblanos presentaron el menor valor $(\mathrm{F}(3,43)=15.74 ; \mathrm{p}<.00)$. En Oaxaca, por otro lado, no hubo diferencias entre el bosque y los sitios MIAF.

Las comunidades fueron predominantemente endogeas tanto en Puebla ( $75 \%$ de las especies) como en Oaxaca (80\%). Tres de las cuatro especies encontradas en
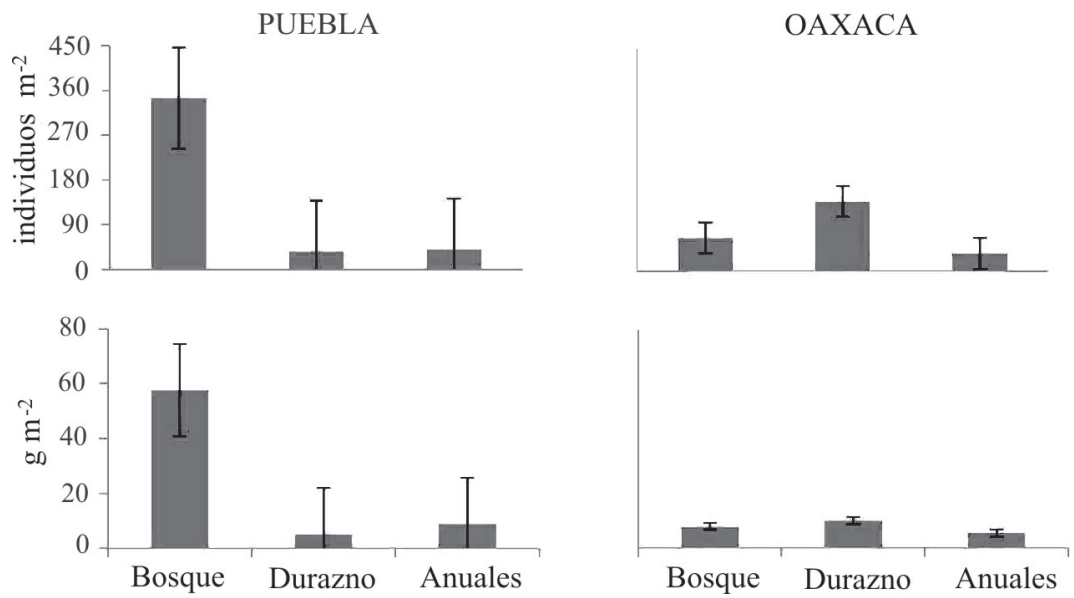

Figura 1. Densidad (individuos $\left.\mathrm{m}^{-2}\right)$ y biomasa $\left(\mathrm{g} \mathrm{m}^{-2}\right)$ de lombrices de tierra encontradas en el bosque $\mathrm{y}$ al interior de los sistemas MIAF en los surcos de durazno y en la parte intermedia dedicada a cultivos anuales de Puebla y Oaxaca. Las líneas en cada barra corresponden el error estándar. 
Puebla fueron típicas de ambientes templados-fríos (L. rubellus, O. tyrtaeum y A. trapezoides). En Oaxaca, por otro lado, tres de las cinco especies encontradas fueron de origen tropical ( $P$. corethrurus, Amynthas sp. y O. occidentalis). Estas especies, además fueron más abundantes en los sitios MIAF que en el bosque; en contraste, las especies de Lumbricidae de climas templados fueron más comunes en el bosque tanto de Puebla como de Oaxaca.

Patrones por especie. Aporrectodea trapezoides. Sólo se encontró en Puebla, tanto en el bosque (50\% de las repeticiones) como en las líneas de duraznos $(25 \%)$ y de anuales (21\%). Estuvo ausente en 13 de los 24 monolitos de los sitios MIAF, aunque su población siempre fue dominante en bosque. Su biomasa representó el $43 \%$ del total de lombrices encontradas.

Dendrodrilus rubidus. Presente solamente en Oaxaca, en el 50\% de las repeticiones del bosque y en las líneas de durazno (17\%) y anuales (17\%). Su biomasa representó solamente $5 \%$ del total.

Lumbricus rubellus. Solamente se encontró en Puebla, preferentemente en el bosque ( $83 \%$ de las repeticiones); en los MIAF sólo se encontró en un monolito (4\%) de los cultivos anuales. La biomasa de esta especie correspondió al $49 \%$ de las lombrices de tierra encontradas.

Octolasion tyrtaeum. Sólo se localizó en el bosque, tanto en Puebla como en Oaxaca, en el 66\% y 17\% de las repeticiones, respectivamente, especialmente en aquellas en donde se presentaron los mayores niveles de humedad. Su biomasa fue del $8 \%$ en Puebla y del 12\% en Oaxaca.

Ocnerodrilus occidentalis. Sólo se encontró en Oaxaca, en el 17\% de las repeticiones del bosque; ausente de las líneas de anuales, se encontró en el $42 \%$ de las repeticiones en las líneas de durazno Su biomasa fue del $2 \%$.

Phoenicodrilus taste. Esta especie sólo se encontró en los sitios MIAF de Puebla, tanto en los duraznos (42\% de las repeticiones) como en las líneas de anuales (33\%). Debido a su pequeño tamaño, su biomasa solamente representó $0.5 \%$ de la biomasa total de lombrices.

Amynthas $s p$. Esta especie sólo se encontró en Oaxaca, en el 17\% de las repeticiones del bosque; en los sitios MIAF ocurrió tanto en las líneas de durazno (50\% de repeticiones) como en las anuales (25\%). Su biomasa fue del $43 \%$.

Pontoscolex corethrurus. Sólo se le encontró en Oaxaca, tanto en el bosque (17\% de las repeticiones) como en las líneas de durazno (58\%) y anuales (58\%) de los sitios MIAF. Su biomasa fue del 33\%.

Por lo tanto, las especies dominantes en Puebla fueron L. rubellus (epigea) y $A$. trapezoides (endogea), que juntas aportaron el $77 \%$ y $92 \%$ de la abundancia y biomasa total, respectivamente. En Oaxaca, las dominantes fueron la epigea Amynthas sp. y la endogea $P$. coretrurus cuya biomasa conjunta representó el $76 \%$ (Cuadro 3 ).

Las comunidades y las condiciones fisicoquímicas del suelo. A nivel regional los 


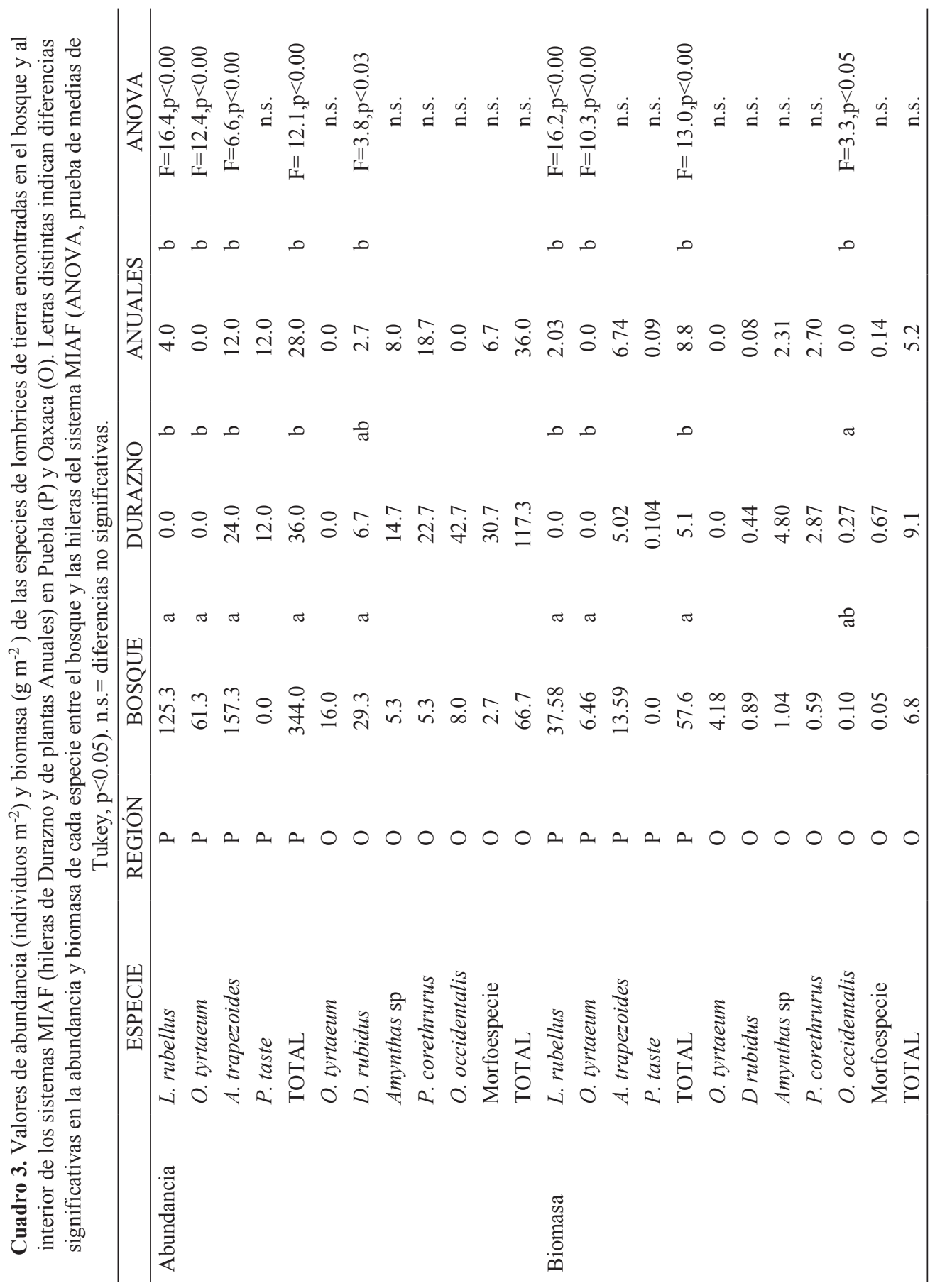


suelos de Oaxaca fueron más ácidos que los de Puebla $(\mathrm{F}(1,20)=24.4,<\mathrm{p}=.00)$, así como más ricos enmateria orgánica $(\mathrm{F}(1,20)=12.7,<\mathrm{p}=.00)$, nitrógeno $(\mathrm{F}(1,20)=27.4,<\mathrm{p}=.00)$ y CIC $(\mathrm{F}(1,20)=15.4,<\mathrm{p}=.00)$ (Cuadro 5). En congruencia con la menor acidez, en Puebla los suelos fueron más ricos en $\operatorname{Mg}(\mathrm{F}(1,20)=29.9,<\mathrm{p}=.00)$.

En Puebla, los bosques fueron mucho más ricos en cationes ( $\mathrm{Ca}, \mathrm{Mg}, \mathrm{CIC}$ ), $\mathrm{MO}$ y N que los suelos cultivados; al interior de los sitios MIAF no hubo diferencias en ninguno de los parámetros edáficos analizados (Cuadro 4). En Oaxaca solamente se observaron diferencias en $\mathrm{K}$ y Mg; sin embargo, los mayores valores se encontraron en la zona de árboles frutales.

En las relaciones significativas $(\mathrm{p}<0.05)$ entre las variables fisicoquímicas y la densidad y biomasa de epigeas y endogeas, se observaron dos patrones importantes con la CIC y la MO. En el bosque de Puebla, se observó una alta correlación positiva

Cuadro 4. Comparación de las variables edáficas en el bosque y al interior de los sistemas MIAF (hileras de Durazno y de plantas Anuales) en Puebla y Oaxaca. Para cada variable letras distintas indican diferencias significativas entre el bosque y las hileras del sistema MIAF (ANOVA, prueba de medias de Tukey, $\mathrm{p}<0.05)$. n.s. $=$ diferencias no significativas.

\begin{tabular}{|c|c|c|c|c|}
\hline & Bosque & Durazno & Anuales & ANOVA \\
\hline \multicolumn{5}{|c|}{ PUEBLA } \\
\hline $\mathrm{pH}$ & 6.3 & 6.4 & 6.1 & n.s. \\
\hline P bray & 6.60 & 24.63 & 15.20 & n.s. \\
\hline K & 1.19 & 0.80 & 0.29 & n.s. \\
\hline $\mathrm{Ca}$ & $9.61 \mathrm{a}$ & $2.99 \mathrm{~b}$ & $2.32 \mathrm{~b}$ & $\mathrm{~F}=59.47 ; \mathrm{p}<0.001$ \\
\hline $\mathrm{Mg}$ & $4.16 \mathrm{a}$ & $2.67 \mathrm{~b}$ & $2.47 \mathrm{~b}$ & $\mathrm{~F}=5.67 ; \mathrm{p}<0.03$ \\
\hline CIC & $13.55 \mathrm{a}$ & $5.67 \mathrm{~b}$ & $5.29 \mathrm{~b}$ & $F=23.68 ; p<0.001$ \\
\hline$\% \mathrm{M} \mathrm{O}$ & $6.12 \mathrm{a}$ & $1.27 \mathrm{~b}$ & $0.88 \mathrm{~b}$ & $\mathrm{~F}=235.2 ; \mathrm{p}<0.001$ \\
\hline$\% \mathrm{~N}$ Total & $0.24 \mathrm{a}$ & $0.07 \mathrm{~b}$ & $0.06 \mathrm{~b}$ & $\mathrm{~F}=92.09 ; \mathrm{p}<0.001$ \\
\hline $\mathrm{C} / \mathrm{N}$ & $15 \mathrm{a}$ & $11.2 \mathrm{~b}$ & $9.38 \mathrm{~b}$ & $\mathrm{~F}=8.42 ; \mathrm{p}<0.001$ \\
\hline \multicolumn{5}{|c|}{ OAXACA } \\
\hline $\mathrm{pH}$ & 4.06 & 5.0 & 4.9 & n.s. \\
\hline P bray & 0.07 & 53.58 & 11.43 & n.s. \\
\hline $\mathrm{K}$ & $0.07 \mathrm{~b}$ & $0.85 \mathrm{a}$ & $0.09 \mathrm{~b}$ & $\mathrm{~F}=9.42 ; \mathrm{p}<0.001$ \\
\hline $\mathrm{Ca}$ & 0.60 & 8.54 & 4.71 & n.s. \\
\hline $\mathrm{Mg}$ & $0.34 \mathrm{~b}$ & $1.82 \mathrm{a}$ & $0.30 \mathrm{~b}$ & $\mathrm{~F}=15.9 ; \mathrm{p}<0.001$ \\
\hline $\mathrm{CIC}$ & 15.18 & 16.11 & 13.21 & n.s. \\
\hline$\%$ M O & 10.46 & 7.45 & 5.91 & n.s. \\
\hline$\%$ N Total & 0.52 & 0.44 & 0.32 & n.s. \\
\hline $\mathrm{C} / \mathrm{N}$ & 11.5 & 9.77 & 9.78 & n.s. \\
\hline
\end{tabular}


Cuadro 5. Abundancia ( $\mathrm{D}$, individuos $\left.\mathrm{m}^{-2}\right)$ y biomasa $\left(\mathrm{B}, \mathrm{g} \mathrm{m}^{-2}\right)$ de las lombrices de tierra al interior de los sistemas MIAF (hileras de Durazno y de plantas Anuales) en Puebla y Oaxaca. Los valores de los sistemas MIAF se presentan por sitio, correspondiendo cada uno a un productor distinto. Para fines comparativos del manejo se incluyen las prácticas de cultivo de cada productor.

\begin{tabular}{|c|c|c|c|c|c|}
\hline \multirow[b]{2}{*}{ PUEBLA } & \multicolumn{2}{|c|}{ Durazno } & \multicolumn{2}{|c|}{ Anuales } & \multirow[t]{2}{*}{ Prácticas de cultivo } \\
\hline & $\mathrm{D}$ & B & $\mathrm{D}$ & B & \\
\hline P1 & 43 & 9.4 & 9 & 0.0 & $\begin{array}{l}\text { Deja crecer y reintegra toda la biomasa herbácea en } \\
\text { durazno. }\end{array}$ \\
\hline $\mathrm{P} 2$ & 85 & 10.9 & 85 & 27.9 & $\begin{array}{l}\text { Hay cultivo de alfalfa y aplica estiércoles } \\
\text { constantemente. }\end{array}$ \\
\hline P3 & 16 & 0.1 & 5 & 0.04 & $\begin{array}{l}\text { Extrae del terreno todos los residuos de cosecha, podas y } \\
\text { herbáceas. }\end{array}$ \\
\hline $\mathrm{P} 4$ & 0 & 0.0 & 32 & 2.1 & Como sistema MIAF está abandonado. No tiene manejo. \\
\hline \multicolumn{6}{|l|}{ OAXACA } \\
\hline P5 & 336 & 5.8 & 32 & 4.2 & $\begin{array}{l}\text { Aplica poco estiércol secado al sol. Los filtros de } \\
\text { escurrimiento tienen MO abundante. }\end{array}$ \\
\hline P6 & 69 & 7.7 & 16 & 3.6 & La sección de cultivos anuales está en descanso. \\
\hline P7 & 107 & 23.4 & 53 & 9.5 & Los filtros de escurrimiento tienen MO abundante. \\
\hline P8 & 53 & 3.1 & 43 & 3.8 & $\begin{array}{l}\text { La sección de cultivos anuales está en descanso. Como } \\
\text { sistema MIAF es poco atendido. }\end{array}$ \\
\hline
\end{tabular}

entre la densidad de lombrices endogeas y la disponibilidad de cationes $(\mathrm{R}=0.99)$; en las líneas de durazno, por otro lado, la densidad de lombrices se correlacionó negativamente con el incremento de la CIC $(\mathrm{R}=-0.88)$; en las áreas dedicadas a cultivos anuales la biomasa de endogeas también se correlacionó negativamente con la CIC $(\mathrm{R}=-0.95)$.

En Oaxaca, la relación más importante se encontró en el incremento de la densidad de lombrices epigeas y la cantidad de $\mathrm{MO}$, tanto en las líneas de durazno $(\mathrm{R}=$ $0.99)$ como en las áreas dedicadas a anuales $(\mathrm{R}=0.96)$.

Patrones al interior del MIAF. Los sitios MIAF de ambas regiones provocaron respuestas muy diferentes en las lombrices de tierra. En Puebla la invasión a las áreas de cultivos anuales parece haber ocurrido desde el bosque, con resultados no muy exitosos y sin un claro patrón dentro de ellos; en Oaxaca, la invasión parece haber ocurrido recientemente hacia el bosque o bien las lombrices se dispersaron con mucho éxito hacia los sitios MIAF, particularmente hacia las líneas de árboles (Cuadro 5).

\section{DISCUSIÓN}

Los sistemas alley cropping (hileras de plantas anuales alternadas con hileras de árboles) han sido diseñados en el mundo para mantener una producción diversificada 
y escalonada a través del año (Midmore 1993, Cortés et al. 2005); para el caso de México, en los sistemas MIAF se han integrado tecnologías en el manejo de nutrientes para incrementar la producción agrícola, aprovechando las diferentes estrategias de absorción que tienen los árboles y las plantas anuales, así como la disposición espacial de las especies que faciliten la captación de la radiación solar.

Tanto las líneas de árboles como las hileras de especies anuales de este sistema benefician al suelo; las primeras aportan hojarasca y sirven de cortavientos, mientras que las segundas fijan $\mathrm{N}_{2}$ atmosférico al suelo cuando las plantas anuales corresponden a leguminosas. Además las líneas de árboles frutales mantienen durante largos periodos de tiempo, una cierta superficie del suelo inalterada por las labores de preparación usadas en las hileras de plantas anuales, lo que las hace un refugio idóneo de poblaciones de macro y microorganismos del suelo. Un beneficio adicional de las hileras de árboles, es que sus raíces logran extraer nutrientes de la profundidad del suelo que, cuando los árboles tiran las hojas, se instaura un sistema de reciclaje dinámico vertical, espacial y temporal.

En un sistema de esta naturaleza la presencia de una comunidad de lombrices de tierra funcionalmente diversa sería un valor agregado al sistema, pues mediante sus actividades podrían incrementar la descomposición de la hojarasca, el reciclaje de nutrientes en el suelo y a la dispersión de los nutrientes desde las hileras de árboles hacia las hileras de anuales.

Los sistemas MIAF de Puebla y Oaxaca mostraron claras diferencias en la comunidad de lombrices, como cabría esperar, dadas las diferencias climáticas y edáficas. La diferencia en precipitación, sin embargo parece que fue el factor que más influencia tuvo sobre las comunidades de lombrices, afectando no solo la diversidad de lombrices en los sistemas MIAF, sino la distribución al interior de las mismas. La diferencia de edades de los sistemas MIAF de cada región (11 años en Puebla y 9 años en Oaxaca), indica que los sistemas más antiguos no necesariamente corresponden a los más diversos.

Comparaciones. Las densidades registradas en los sistemas MIAF de Puebla (36-40 individuos $\mathrm{m}^{-2}$ ) y de Oaxaca (36-141 individuos $\mathrm{m}^{-2}$ ) fueron similares a las encontradas en otros sistemas agroforestales de clima templado: en Ohio, USA 10-350 individuos $\mathrm{m}^{-2}$ (Bohlen et al. 1995); en Ontario y Quebec Canadá 10-180 individuos $\mathrm{m}^{-2}$ (Price y Gordon 1999), y 46-253 individuos $\mathrm{m}^{-2}$ (Whalen 2004), respectivamente. En estos trabajos se encontraron además, al menos algunas de las especies registradas en este estudio

La presencia de lombrices de afinidad más tropical en la región de Oaxaca ( $O$. occidentalis y $P$. corethrurus) refleja la respuesta de las lombrices de tierra a los cambios de altitud y de latitud, pero principalmente el efecto de la temperatura. Los quinientos metros de diferencia en la altitud de esta región y la de Puebla, además de un grado de menor latitud, ciertamente repercute en la invasión de lombrices tropica- 
les, a pesar de que la diferencia en la temperatura media anual fue solo de $1.5^{\circ} \mathrm{C}$. Las temperaturas mínimas, sin embargo son una explicación más clara para este patrón pues en Puebla baja hasta los $1.2^{\circ} \mathrm{C}$ en época invernal, mientras que en Oaxaca lo hace solamente a $8.4^{\circ} \mathrm{C}$.

Restricciones de humedad. En Puebla el bosque presentó una mayor densidad y biomasa de lombrices que los sistemas MIAF, mientras que en Oaxaca estas diferencias no fueron significativas. En puebla este contraste entre el ecosistema natural y los sistemas manejados se debe muy probablemente a la menor humedad existente de los sistemas MIAF; por su mayor humedad el bosque aledaño estaría funcionando como un refugio para estos organismos. De hecho la especie más común del bosque y la de mayores valores en los sistemas MIAF (A. trapezoides), ha sido reconocida como una especie tolerante a la sequía (Bohlen et al. 1995; Chan \& Mead 2003; Fernández et al. 2010). La otra especie común en el bosque de Puebla fue la epigea L. rubellus, que abundó sobre todo cerca de cuerpos de agua. Esta especie estuvo prácticamente ausente en los sistemas MIAF, pues sólo se encontró en un sitio MIAF que se caracterizó por el uso constante de estiércol de bovino ( $8.3 \%$ de las repeticiones totales).

Aunque se ha demostrado que en función de las condiciones de humedad, L. rubellus puede actuar como una epiendogea (Addison 2009), en Puebla su comportamiento en el sistema MIAF correspondió al de una epigea restringida, pues un mes después de la última lluvia del año las lombrices recién-eclosionadas se encontraron muertas y secas. De modo que el mantenimiento de poblaciones viables de esta especie en los sistemas MIAF solamente ocurrirá si la humedad aumenta, ya sea por irrigación artificial o por un sistema de cobertura que proteja la humedad del suelo por más tiempo, como en el bosque.

O. tyrtaeum fue otra especie del bosque de Puebla y Oaxaca que estuvo completamente ausente de los sistemas MIAF. Esto podría deberse a una muy baja tolerancia a la sequía, ya que se trata de una especie frecuente y bien adaptada a suelos muy húmedos (Hendrix et al. 2008) y pantanos (Plum \& Filser 2005); en el bosque de Puebla, por ejemplo, se le encontró en los flujos de agua de los arroyos. Su introducción al sistema MIAF estaría también fuertemente condicionada por la humedad; sin embargo, y debido a que se ha registrado como una especie poco tolerante a la manipulación (García \& Fragoso 2002) su sobrevivencia en los sistemas MIAF podría no depender únicamente de la humedad.

Finalmente en Puebla, solamente $P$. taste pudo soportar el clima y las labores agrícolas que se aplican en los sistemas MIAF, pues estuvo presente en 11 de los 24 monolitos efectuados en los cuatro sistemas MIAF (45.8\%). Se trata de una especie con una plasticidad ecológica alta y muy común en los trópicos de todo el mundo (Fragoso et al. 1999) y que en México ha sido registrada en áreas de clima árido con riego (Brito-Vega et al. 2006). Por su pequeño tamaño, sin embargo, no sería una especie con efectos importantes en la fertilidad de los sistemas MIAF. 
Con poco más del doble de precipitación anual en Oaxaca que en Puebla, los sistemas MIAF de Oaxaca, no tuvieron una limitación importante de humedad para las lombrices de tierra. En particular esto se observó para las tres especies tropicales ( $P$. corethrurus, Amynthas sp. y $O$. occidentales), pues las especies de afinidad templada (O. tyrtaeum y D. rubidus) fueron más abundantes en el bosque. Por su gran tolerancia ambiental (especies euri, Fragoso 2001), las tres especies tropicales tienen una elevada potencial de mantenerse en los sistemas MIAF y una de ellas ( $P$. corethrurus) inclusive, se ha demostrado que aumenta la producción agrícola en diferentes tipos de cultivos (Brown et al. 1999).

Materiales orgánicos. En Puebla, las condiciones extremas de baja humedad y la intensificación agrícola utilizada, no permitieron evaluar el efecto del aporte de hojarasca al interior del sistema MIAF; en Oaxaca, sin embargo, el efecto se observó claramente bajo los árboles por una mayor abundancia y biomasa total de lombrices y de las tres especies más abundantes. Este efecto sería el resultado de las acumulaciones de materiales orgánicos en los filtros de escurrimiento y de una reducida extracción de residuos de cosecha. Tal y como ha sido ampliamente señalado, una cobertura vegetal favorecerá la presencia y abundancia de lombrices (Kalisz \& Powell 2000; Morales \& Sarmiento 2002); las epigeas en particular, serán favorecidas por materiales altamente palatables (Chaudhuri et al. 2008).

Relación con las variables fisicoquímicas. La alta correlación en el bosque de Puebla entre la abundancia de lombrices endogeas y la CIC, podría estar indicando el efecto de las lombrices de tierra en la descomposición de la hojarasca y el reciclamiento de nutrientes. En los sistemas MIAF, en donde no hubo relación entre la CIC y las lombrices, es posible que esta relación haya quedado enmascarada por la aplicación constante de fertilizantes.

La alta abundancia y biomasa de lombrices en los bosques de Puebla puede también ser el resultado de suelos más ricos en nutrientes $(\mathrm{Ca}$ y $\mathrm{Mg}$ ) coincidiendo con otros resultados obtenidos en el sureste de México (Huerta et al. 2008). Siendo la materia orgánica-hojarasca y los nutrientes del suelo el alimento principal de, respectivamente, las epigeas y de las endogeas, la relación epigeas/endogeas permite conocer que la comunidad de lombrices de tierra estará determinada por la cantidad de materia orgánica o por los nutrientes del suelo (Falco \& Momo 2010). En el bosque de Puebla, en donde el suelo contiene un $6.1 \%$ de MO, la relación densidad de epigeas/densidad de endogeas fue $1 / 1.7=0.57$, mientras que en el bosque de Oaxaca, en donde el suelo contiene un $10.4 \%$ de MO, la relación fue de casi el doble (1/0.8 $=1.18$ ). En contraste, en los sistemas MIAF de Puebla, cuyos valores de MO fueron mucho menores $(1.08 \%)$, esta relación fue considerablemente menor $(1 / 15=0.07)$, mientras que en los sistemas MIAF oaxaqueños, con un $6.4 \%$ de MO, la relación fue cercana a los bosques de Puebla $(1 / 2.6=0.38)$.

Bajo esta perspectiva los sistemas MIAF de Oaxaca pueden considerarse con con- 
diciones ecológicas similares al bosque de Puebla, mientras que el suelo de los sistemas MIAF poblanos tendrían las condiciones más adversas para el desarrollo de las comunidades de lombrices de tierra.

Manejo en el MIAF. Las comunidades de lombrices de tierra de ambientes manejados se estructuran principalmente por la interacción de las variables ambientales (temperatura, humedad, cobertura vegetal, nutrientes, etc.), con el manejo antropogénico. Todas estas variables están jerárquicamente relacionadas, de tal modo que aunque la mayoría presenten los valores adecuados para el mantenimiento de las lombrices de tierra, si una variable de un nivel jerárquico superior tiene valores inadecuados, es poco probable que se mantengan poblaciones viables. Por ejemplo si la vegetación (multi-estrato) no ofrece un refugio a las condiciones de baja humedad, las lombrices de tierra no podrán subsistir.

En Puebla, la cobertura y hojarasca producida por los árboles de durazno en los sistemas MIAF estudiados aparentemente no fue suficiente para impedir la pérdida de agua y mantener así una comunidad diversa y abundante de lombrices. Sin embargo es altamente probable que con la aplicación de estiércol, la reincorporación abundante de biomasa herbácea y la disminución de labores agrícolas, se podría lograr que el sistema albergara una mayor cantidad de lombrices. Feijoo et al. (2007), por ejemplo, reconocen que hay sistemas de cultivo que crean ambientes propicios para conservar la biodiversidad, la recolonización y la reproducción natural de las especies. En los sistemas MIAF estudiados y especialmente en Puebla, se observó una alta remoción del suelo y de la cubierta vegetal, que además de afectar la fertilidad del suelo, afecta negativamente la humedad del suelo, cuya temporalidad y frecuencia determinan ya una intensificación del cultivo (Postma-Blaauw et al. 2010).

Con base en otras experiencias en cultivos perennes (Senapati et al. 1999) o con anuales (Li et al. 2002), la presencia de lombrices, tanto en las zonas bajo el dominio de los árboles como de las plantas anuales, deberá tener un efecto positivo y sinérgico sobre la producción. Brown et al. (1999) sugieren que una biomasa de lombrices cercana o similar a los $30 \mathrm{~g} \mathrm{~m}^{-2}$ es suficiente para impactar significativamente la fertilidad del suelo. Sin embargo valores cercanos a esta biomasa, sólo se observaron en la hilera de anuales de un sistema MIAF de Puebla (P2, $\left.27.9 \mathrm{~g} \mathrm{~m}^{-2}\right)$ sometido a prácticas de fertilización natural con estiércol y en la hilera de árboles de otro sistema MIAF de Oaxaca (P7, $23.3 \mathrm{~g} \mathrm{~m}^{-2}$ ). Esto indica que el sistema MIAF puede verse altamente beneficiado si se logra incrementar la cantidad y biomasa de lombrices, lo que se podrá alcanzar mediante la manipulación de la humedad, de los residuos orgánicos producidos y una cierta fertilización orgánica con estiércol.

\section{CONCLUSIONES}

Los sistemas MIAF son sistemas agrícolas diseñados para diversificar e incrementar la producción agrícola; la combinación entre especies perennes y anuales permite 
conformar una estructura vegetal ordenada en el espacio y tiempo, que constituye un refugio potencial para las comunidades edáficas. Sin embargo el efecto sobre la fauna del suelo podrá variar, dependiendo de las condiciones específicas de cada lugar.

Tanto en Puebla como en Oaxaca, el suelo de las líneas de árboles frutales de los sistemas MIAF no se distinguió como un refugio para la preservación de las comunidades de lombrices de tierra, a pesar de mantener niveles bajos de remoción del suelo $\mathrm{y}$ de su condición agroforestal.

Con objeto de incrementar las poblaciones de lombrices, y su consecuente efecto benéfico adicional en el suelo, en Puebla es más necesaria la manipulación de los sistemas MIAF, sobre todo para implementar cambios que aumenten la cantidad y conservación de la humedad y de la materia orgánica. Entre estas prácticas se propone el uso de abonos de cobertera, estiércol, etc., que ayuden a proteger el suelo, aumentar la cantidad de hojarasca y la humedad del suelo, así como incrementar la mineralización y liberación de nutrientes para las plantas.

Finalmente, y con base en los resultados aquí presentados, las siguientes especies de lombrices de tierra serían susceptibles de manejo para mejorar la fertilidad en los sistemas MIAF: L. rubellus (epigea) y A. trapezoides (endogea) en Puebla y Amynthas sp. (epigea), D. rubidus (epigea) y P. corethrurus, (endogea), en Oaxaca.

AGRADECIMIENTOS. Dionicio Juárez (DJ) agradece al Centro de Agroecología del Instituto de Ciencias y a la BUAP, por su apoyo para llevar a cabo los estudios de posgrado así como al Colegio de Postgraduados por la formación durante los estudios de doctorado y por comprender la importancia de la biota edáfica para el desarrollo agrícola. DJ también agradece al CONAC YT, por la beca otorgada para realizar esta investigación como parte de la obtención del grado de doctor. Los dos autores agradecen a los campesinos de Puebla y Oaxaca por haber permitido llevar a cabo el estudio en sus parcelas productivas. También se agradece a las técnicas Ninfa Portillo y Sandra Rocha del LAAR del INECOL por su apoyo en los análisis de suelos. Finalmente agradecemos las correcciones de dos revisores anónimos, cuyas atinadas sugerencias ayudaron a mejorar considerablemente este trabajo.

\section{LITERATURA CITADA}

Addison, J. A. 2009. Distribution and impacts of invasive earthworms in Canadian forest ecosystems. Biol Invasions 11:59-79.

Anderson, J. M. \& Ingram, J. S. I. 1993. Tropical soil biology and fertility: a handbook methods. C.A.B.I. Wallingford, Oxford, U.K.

Blakemore, R. J. 2006. Cosmopolitan Earthworms - an Eco-Taxonomic Guide to the Peregrine Species of the World. VermEcology, Japan.

Bohlen, P. J., Edwards, W. M. \& Edwards, C. A. 1995. Earthworm community structure and diversity in experimental agricultural watersheds in Northeastern Ohio. Plant Soil 170: 233-239.

Brito-Vega, H., Espinosa-Victoria, D., Figueroa-Sandova, B., Fragoso, C. \& Patrón-Ibarra, J. C. 2006. Diversidad de lombrices de tierra con labranza de conservación y convencional. Terra Latinoamericana 24: 99-108.

Brown, G., Pashanasi, B., Villenave, C., Patrón, J. C., Senapati, B. K., Giri, S., Barois, I., Lavelle, P., Blanchart, E., Blakemore, R. J., Spain, A. V.\& Boyer, J. 1999. Efects of earthworms on plant 
production in the tropics, pp. 87-147. In: Lavelle P., Brussaard L., Hendrix P. (Eds.). Earthworm management in tropical agroecosystems. C.A.B.I. Wallingford, Oxford, U.K.

Chan, K. Y. \& Mead, J. A. 2003. Soil acidity limits colonisation by Aporrectodea trapezoides, an exotic earthworm. Pedobiologia, 47: 225-229.

Chaudhuri. P. S, Nath, S. \& Paliwal, R. 2008. Earthworm population of rubber plantations (Hevea brasiliensis) in Tripura, India. Tropical Ecology, 49: 225-234.

Conover, W. J. \& Iman, R. L. 1981. Rank transformations as a bridge between parametric and nonparametric statistics. American Statistician, 35: 124-129.

Cortés F., J.I., Mendoza R., R., Hernández R., E., Aceves R., E., Turrent F., A. \& Estrella C., N. 2004. El sistema agrícola "Milpa intercalada en árboles frutales (MIAF)" en terrenos planos. Colegio de Postgraduados, Puebla, México.

Cortés F., J.I., Turrent F., A., Díaz V., P., Hernández R., E., Mendoza R., R. \& Aceves R., E. 2005. Manual para el establecimiento y manejo del sistema milpa intercalada con árboles frutales (MIAF) en laderas. Colegio de Postgraduados, México.

Falco, L. B. \& Momo, F. 2010. Selección de hábitat: efecto de la cobertura y tipo de suelo en lombrices de tierra. Acta Zoológica Mexicana (n.s.), Número especial 2: 179-187.

Feijoo, A., Zúñiga, M. C., Quintero, H. \& Lavelle, P. 2007. Relaciones entre el uso de la tierra y las comunidades de lombrices en la cuenca del río La Vieja, Colombia. Pastos y Forrajes, 30: 235249.

Fernández, R., Novo, M., Gutiérrez, M., Almodóvar, A. \& Díaz C., D.J. 2010. Life cycle and reproductive traits of the earthworm Aporrectodea trapezoides (Duges, 1828) in laboratory cultures. Pedobiologia, 53: 295-299.

Fragoso, C. 2001. Las lombrices de tierra de México (Annelida, Oligochaeta): Diversidad, ecología y manejo. Acta Zool. Mex. Special number 1: 131-171.

Fragoso C. \& Rojas, P. 2014. Biodiversidad de lombrices de tierra (Annelida: Oligochaeta: Crassiclitellata) en México. Revista Mexicana de Biodiversidad, Supl. 85: S197-S207.

García, E. 1973. Modificaciones al sistema de clasificación climática de Koppen; para adaptarlo a las condiciones de la República Mexicana. UNAM, México.

García, J. A. \& Fragoso, C. 2002. Growth, reproduction and activity of earthworms in degraded and amended tropical open mined soils: laboratory assays. Applied Soil Ecology 20: 43-56 .

Hendrix, P. F. \& Edwards, C. A. 2004. Earthworms in Agroecosystems: Research Approaches, pp. 287-296. In: C.A. Edwards (ed). Earthworm Ecology. CRC Press. Boca Raton. Florida.

Hendrix, P. F., Callaham Jr., M. A., Drake, J. M., Huang, C., James, S. W., Snyder, B. A. \& Zhang, W. 2008. Pandora's Box Contained Bait: The Global Problem of Introduced Earthworms. Annual Review of Ecology and Systematics, 39: 593-613.

Huerta L. E., Rodríguez-Olán, J., Evia-Castillo, I., Montejo-Meneses, E., Cruz-Mondragón, M. \& García-Hernández, R. 2008. Relación entre la fertilidad del suelo y su población de macroinvertebrados. Terra Latinoamericana 26: 171-181.

Kalisz, P. J. \& Powell E. J. 2000. Invertebrate macrofauna in soils under old growth and minimally disturbed second growth forests of the Appalachian mountains of Kentucky. The American Midland Naturalist, 44: 297-307.

Lavelle, P., Barois, I., Blanchart, E., Brown, G.G., Brussaard, L., Decaëns, T., Fragoso, C., Jiménez, J.J., Ka Kajondo, K., Martínez, M.A., Moreno, A.G., Pashanasi, B., Senapati, B.K. \& Villenave, C. 1998. Earthworms as a resource in tropical agroecosystems. Nature \& Resources 34: 26-41.

Lavelle, P., Brussaard, L. \& Hendrix, P. 1999. Earthworm management in tropical agroecosystems. C.A.B.I. Wallingford, Oxford, U.K. 
Li, H., Hu, F., Shen, Q., Chen, X., Cang, L. \& Wang, X. 2002. Effect of earthworm inoculation on soil carbon and nitrogen dynamics and on crop yield with application of corn residues. Chinese Journal of Applied Ecology, 13:1637-1641

Midmore, D. J. 1993. Agronomic modification of resource use and intercrop productivity. Field Crops Research, 3-4: 357-380.

Mijangos, I., Pérez, R., Albizu, I. \& Garbisu, C. 2006. Effects of fertilization and tillage on soil biological parameters. Enzyme and Microbial Technology, 40: 100-106.

Montoya, J. M. \& Solé A., R. 2002. Small World Patterns in Food Webs. The Journal of Theoretical Biology, 214: 405-412.

Morales J. \& Sarmiento, L. 2002. Dinámica de los macroinvertebrados edáficos y su relación con la vegetación en una sucesión secundaria en el páramo venezolano. Ecotrópicos, 1: 99-110.

Plum, N. M. \& Filser, J. 2005. Floods and drought: Response of earthworms and potworms (Oligochaeta: Lumbricidae, Enchytraeidae) to hydrological extremes in wet grassland. Pedobiologia, 49: 443-453.

Postma-Blaauw, M. B., de Goede, R. G. M, Bloem, J., Faber, J. H. \& Brussaard, L. 2010. Soil biota community structure and abundance under agricultural intensification and extensification. Ecology, 91: 460-473.

Price, G. W. \& Gordon, A. M. 1999. Spatial and temporal distribution of earthworms in a temperate intercropping system in southern Ontario, Canada. Agroforestry Systems, 44: 141-149.

Reynolds, J. W. 1977. The Earthwomrs (Lumbricidae and Sparganophiidae) of Ontario. Royal Ontario Museum, Toronto, Canada.

Senapati, B. K., Lavelle, P., Panigrahi, P .K., Giri, S. \& Brown, G. G. 1999. Case study A1. Soil Fauna and organic fertilizers in tea gardens of Tamil Nadu, India. FAO. Disponible en: http://www. fao.org/landandwater/agll/soilbiod/cases/caseA1.pdf

Torres Z., J. P., Cortés F., J. I., Turrent F., A., Hernández R., E. \& Muratalla L., A. 2008. Rendimiento de fruto y número de ramas principales en árboles de durazno intercalados con milpa. Terra Latinoamericana, 26: 265-273.

Werner, M. R. 1990. Earthworm ecology and sustaining agricultural. Components 4(1). Disponible en: http://www.sarep.ucdavis.edu/worms/werner.htm.

Whalen, J. K. 2004. Spatial and temporal distribution of earthworm patches in corn field, hayfield and forest systems of southwestern Quebec, Canada. Applied Soil Ecology, 227: 143-151.

Zar, J. H. 2009. Biostatistical analysis. Prentice \& Hall. USA. 